\title{
Clinicopathological significance of cytoplasmic transducer of ErbB2. 1 expression in gastric cancer
}

\author{
SU-QING ZHANG ${ }^{1 *}$, KE-KANG SUN ${ }^{2 *}$, XIAO-YANG WU ${ }^{2}$, NING ZHONG $^{2}$, HUA ZHAO $^{1}$ and DE-CHUN LI ${ }^{1}$ \\ ${ }^{1}$ Department of General Surgery, First Hospital Affiliated to Soochow University, Suzhou, Jiangsu 215006; \\ ${ }^{2}$ Department of Gastrointestinal Surgery, Kunshan First People's Hospital Affiliated to Jiangsu University, \\ Kunshan, Jiangsu 215300, P.R. China
}

Received May 26, 2014; Accepted February 17, 2015

DOI: $10.3892 / \mathrm{mmr} .2015 .3470$

\begin{abstract}
The aim of the present study was to investigate the expression of transducer of ErbB2.1 (TOB1) in gastric carcinoma and to clarify the association between TOB1 expression and the clinical significance of this expression in patients with gastric carcinoma. Western blot analysis was performed to confirm the expression of TOB1 in gastric cancer. Immunohistochemistry (IHC) was performed on a tissue microarray containing 90 pairs of primary gastric cancer and adjacent normal tissue samples. TOB1 expression was evaluated separately with cytoplasmic and nuclear staining. Western blot analysis revealed significantly lower expression levels of TOB1 in gastric cancer tissues than those in adjacent normal tissues in $91.7 \%$ of cases. This was confirmed by IHC, which revealed decreased cytoplasmic TOB1 expression in cancer tissues compared with those of normal tissue samples in $84.4 \%$ of cases. The IHC data also revealed low cytoplasmic expression of TOB1 in $67.8 \%$ of human gastric cancer samples. Nuclear TOB1 expression exhibited no significant association with specific pathological features. However, a significant association was identified between cytoplasmic expression levels of TOB1 and clinicopathological characteristics, including the depth of invasion $(\mathrm{P}=0.017)$, differentiation grade $(\mathrm{P}=0.034)$ and tumor-node-metastasis stage $(\mathrm{P}<0.000)$. In conclusion, cytoplasmic TOB1 expression was suggested to be significant in angiogenesis and cell differentiation in gastric cancer tissues and may be used as a potential prognostic marker.
\end{abstract}

\section{Introduction}

Globally, gastric cancer is the fourth most common type of cancer and the second leading cause of cancer-associated

Correspondence to: Dr De-Chun Li, Department of General Surgery, First Hospital Affiliated to Soochow University, 96 Shizi Road, Suzhou, Jiangsu 215006, P.R. China

E-mail: lidechun12345@126.com

${ }^{*}$ Contributed equally

Key words: transducer of ErbB2. 1, gastric cancer, tissue microarray mortality $(1,2)$. Although, the current treatment protocol for gastric cancer incorporates chemotherapy or radiotherapy with surgical resection, the survival rate of patients with gastric cancer has remained low $(1,3,4)$. Considering the high level of incidence and the high mortality rate of patients with gastric cancer, elucidation of the biological basis of tumor development and progression is required in order to develop markers for assessing disease onset or to predict the outcome of therapeutics, as well as to identify targets for the development of novel therapies (5-7).

Overexpression of human epidermal growth factor receptor 2 (ErbB2), also known as Her2, which is a membrane-associated tyrosine kinase, may result in the activation of certain cellular signal transduction systems (8). Numerous studies have observed an association between Her2 overexpression and invasion, metastasis and poor prognosis of patients with gastric cancer $(7,9,10)$. The transducer of ErbB2. 1 (TOB1) gene was initially identified as a member of the anti-proliferative transducer of ErbB2/B-cell translocation gene (TOB/BTG) protein family, which was identified in 1990 (11). Multiple lines of evidence have demonstrated that TOB1 is able to inhibit cell proliferation through its interventions in oncogenic pathways, involving endocytic mechanisms and cellular trafficking processes, which were observed to be in a molecular complex with ErbB2 $(12,13)$. To clarify the association between TOB1 expression and clinical outcome, a tissue microarray analysis was performed on samples from patients with gastric cancer.

In the present study, the clinicopathological role of TOB1 expression in gastric cancer was investigated using immunostaining and western blot analysis of clinical specimens from patients with gastric cancer. The association between TOB1 expression and clinical outcome was also evaluated. The present study aimed to elucidate the mechanism underlying the function of TOB1 in the tumorigenesis of gastric cancer, as well as the potential of TOB1 targeted therapy for the treatment of patients with gastric cancer.

\section{Patients and methods}

Patients and specimens. Primary samples of gastric cancer tissue and corresponding noncancerous tissues were obtained during surgery from 12 patients (nine male, 3 female; Age 
range, 47-83 years; Average age, 65 years; Tumor differentation grade II or III) who were treated at The Kunshan First People's Hospital Affiliated to Jiangsu University (Kunshan, China) between September 2013 and December 2013. No patients had received chemotherapy or radiotherapy prior to the surgery. The materials to be analyzed were assessed by a pathologist to ensure that the samples were macroscopically entirely cancerous and selected from an area free from necrotic tissue. Cancerous and noncancerous tissue samples were stored at $-80^{\circ} \mathrm{C}$ prior to protein extraction.

Ethical statement. The present study was undertaken under the guidelines of the ethics committee of Jiangsu University (Zhenjiang, China). Written informed consent was obtained from all patients prior to enrollment in the study. The procedure of collecting human gastric cancer resection specimens from the patients conformed to the principles outlined in the Declaration of Helsinki in 1964.

Western blot analysis. Cell lysates were prepared and western blot analysis was performed as previously described (14). Equal samples of total cell protein $(50 \mu \mathrm{g}$ per lane) were electrophoresed on 10\% SDS-polyacrylamide gels (EMD Millipore, Billerica, MA, USA), transferred onto polyvinylidene difluoride membranes (EMD Millipore) and then blotted using a primary antibody specific for TOB1 (E-1; Santa Cruz Biotechnology, Inc., Dallas, TX, USA; 1:500) at $4^{\circ} \mathrm{C}$ overnight. The blots were subsequently rinsed in $0.1 \%$ Tris-buffered saline-Tween 20 (Shanghai Biological Engineering Co., Ltd., Shanghai, China) and further incubated at $4^{\circ} \mathrm{C}$ for $2 \mathrm{~h}$ with a peroxidase-conjugated anti-mouse secondary antibody (1:2,000; cat. no. sc-133095; Santa-Cruz Biotechnology, Inc.). The protein bands were visualized using an enhanced chemiluminescence system (Union Bioscience Corporation, Hangzhou, China) and exposed to film (Kodak aerial films; Kodak, Rochester, NY, USA). A mouse monoclonal antibody to $\beta$-actin (Santa Cruz Biotechnology, Inc.; 1:3,000) was used as the gel loading control. The densitometry of the protein bands was quantified using Quantity One v4.4.0.36 software (Bio-Rad Laboratories, Inc., Hercules, CA, USA). At least three independent experiments were performed for each cell type investigated.

Tissue microarrays. A tissue microarray (HStmAde180CS-01), purchased from Shanghai Superchip Co., Ltd. (Shanghai, China), was performed (15). All cases were histologically reviewed using hematoxylin and eosin (H\&E) staining. The H\&E sections were examined by two independent pathologists using a light microscope (Olympus, Tokyo, Japan). Duplicate 1-mm diameter cylinders from the tumor centre were selected from each sample, together with paracancerous nonmalignant gastric tissues as controls, to ensure reproducibility and homogenous staining of the slides. Pathological diagnosis, grade and stage were retrieved from the patient's medical records. For all 90 pairs of gastric cancer tissues (Shanghai Zhongshan Hospital; Shanghai Cancer Hospital; Shanghai Ruijin Hospital; Shanghai Renji Hospital) and the corresponding normal tissues obtained from the same patients, 180 cores were applied per slide.
Table I. Clinicopathological data of the 90 patients with gastric cancer analyzed.

\begin{tabular}{|c|c|c|}
\hline Characteristic & No. of patients & $\%$ \\
\hline \multicolumn{3}{|l|}{ Gender } \\
\hline Male & 65 & 72.2 \\
\hline Female & 25 & 27.8 \\
\hline \multicolumn{3}{|l|}{ Age (years) } \\
\hline$<60$ & 37 & 41.1 \\
\hline$\geq 60$ & 53 & 58.9 \\
\hline \multicolumn{3}{|c|}{ Tumor size (mm) } \\
\hline$<40$ & 20 & 22.2 \\
\hline$\geq 40$ & 70 & 77.8 \\
\hline \multicolumn{3}{|c|}{ Depth of invasion } \\
\hline pT1 & 7 & 7.8 \\
\hline pT2 & 17 & 18.9 \\
\hline pT3 & 47 & 52.2 \\
\hline pT4 & 19 & 21.1 \\
\hline \multicolumn{3}{|l|}{ Nodal status } \\
\hline pNo & 31 & 34.4 \\
\hline $\mathrm{pN} 1$ & 12 & 13.3 \\
\hline $\mathrm{pN} 2$ & 18 & 20.0 \\
\hline $\mathrm{pN} 3$ & 29 & 32.3 \\
\hline \multicolumn{3}{|l|}{ Metastasis } \\
\hline M0 & 80 & 88.9 \\
\hline M1 & 10 & 11.1 \\
\hline \multicolumn{3}{|c|}{ Differentiation grade } \\
\hline Grade I & 3 & 3.3 \\
\hline Grade II & 50 & 55.6 \\
\hline Grade III & 37 & 41.1 \\
\hline \multicolumn{3}{|l|}{ TNM stage } \\
\hline I & 15 & 16.7 \\
\hline II & 27 & 30.0 \\
\hline III & 38 & 42.2 \\
\hline IV & 10 & 11.1 \\
\hline \multicolumn{3}{|c|}{ TOB1 in cytoplasm } \\
\hline Low & 61 & 67.8 \\
\hline High & 29 & 32.2 \\
\hline \multicolumn{3}{|c|}{ TOB1 in nucleus } \\
\hline Low & 41 & 45.6 \\
\hline High & 49 & 54.4 \\
\hline
\end{tabular}

TNM, tumor-node-metastasis; TOB1, transducer of ErbB2. 1.

Immunohistochemistry (IHC). IHC was performed using the standard streptavidin-biotin-peroxidase complex method as described previously (16). Tissue microarray slides were incubated at $4^{\circ} \mathrm{C}$ in a humidified chamber overnight with the aforementioned primary antibody specific for TOB1 $(1: 1,000)$. Phosphate-buffered saline (Union Bioscience Corporation, Hangzhou, China) was used instead of the primary antibody against TOB1 as a negative control. Expression of TOB1 was determined in the nucleus and cytoplasm by semi-quantitative 
A

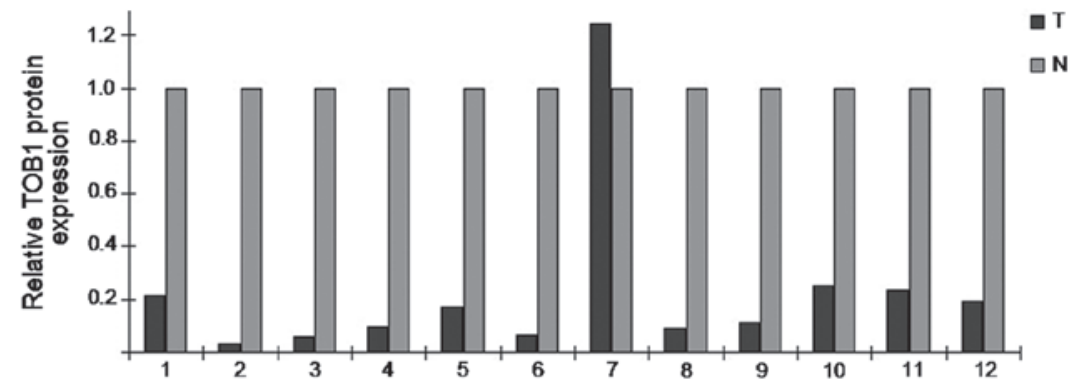

B

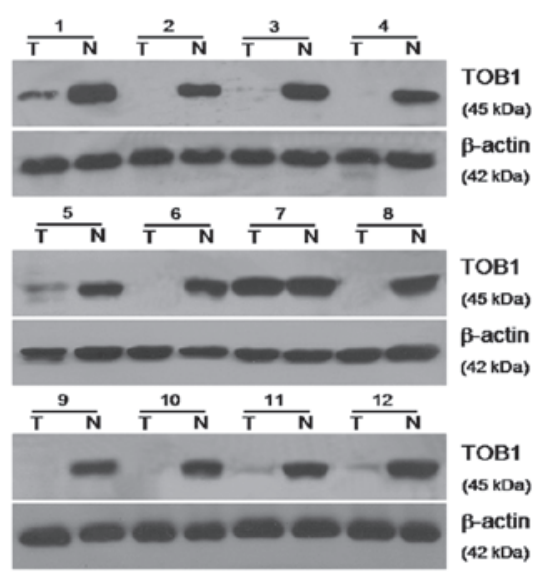

C

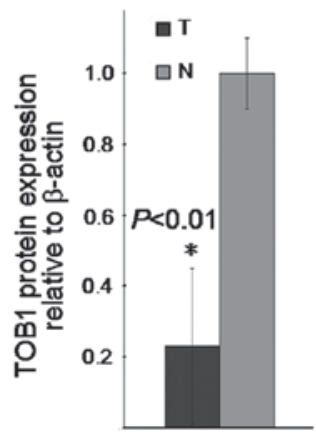

Figure 1. TOB1 expression in gastric cancer and corresponding normal tissues. (A) Expression levels of TOB1 protein were downregulated in 11 out of 12 gastric cancer tissue samples compared with those of the corresponding normal tissue. Relative TOB1 protein expression is displayed in the histogram. (B) Western blot analysis revealed that TOB1 protein expression was downregulated in 11 out of 12 gastric cancer samples. $\beta$-actin was used as the loading control. (C) Average relative protein expression levels of TOB1 in gastric cancer tissues compared with the corresponding normal tissues (n=12). TOB1, transducer of ErbB2. 1; T, primary gastric cancer tissues; N, corresponding normal tissues.

assessment of the percentage of marked tumor cells and the staining intensity. The expression levels of TOB1 were determined by integrating the percentage of positive tumor cells and the intensity of positive staining. The intensity of the staining was scored as follows: Negative (score 0), weak (score 1) or high (score 2). The staining extent was scored according to the percentage of positively stained tumor cells in the field: Negative (score 0), $0-50 \%$ (score 1) or $51-100 \%$ (score 2). The product of the intensity and extent score was considered as the overall IHC score (0-4). The staining was observed and assessed by two independent pathologists blinded to the properties of the samples. The scores for the percentage of positive cells and the scores for expression intensities were multiplied to calculate an immunoreactive score (IRS). An IRS of 0 or 1 was considered to indicate low expression, and an IRS of 2-4 was considered to indicate high expression.

Statistical analysis. Statistical analysis was performed using the SPSS statistical software package (SPSS version 17.0; SPSS, Inc., Chicago, IL, USA). The association between TOB1 protein expression and the clinicopathological features was analyzed using the $\chi^{2}$ test. $\mathrm{P}<0.05$ was considered to indicate a statistically significant difference.

\section{Results}

Expression of TOB1 is decreased in gastric cancer tissues. To examine the expression of TOB1 in gastric cancer tissues, the protein levels were detected in 12 paired primary gastric cancer tissues and the corresponding adjacent normal tissues using western blotting. In $91.7 \%$ (11/12) of specimens, TOB1 expression was reduced in the cancerous tissues compared with that of the noncancerous tissues (Fig. 1A and B). As shown in Fig. 1C, this reduction was statistically significant $(\mathrm{P}<0.01)$. These data confirmed that reducing TOB1 expression is an important event in gastric tumorigenesis.

Patient characteristics. Clinicopathological characteristics of the 90 patients are shown in Table I. The samples included $65(72.2 \%)$ male and $25(27.8 \%)$ female patients with gastric cancer. A total of $37(41.1 \%)$ patients were $<60$ years old and the remaining 53 patients were $\geq 60$ years old. The tumor size of $20(22.2 \%)$ patients was $<40 \mathrm{~mm}$ and that of the remaining patients was $\geq 40 \mathrm{~mm}$. A total of $59(65.6 \%)$ patients exhibited lymph node metastasis and 15 (16.7\%) tumors were categorized as T1, $27(30.0 \%)$ as T2, $38(42.2 \%)$ as T3 and $10(11.1 \%)$ as $\mathrm{T} 4$ according to the American Joint Committee on Cancer (17). Subsequently, TOB1 expression was examined using IHC. In each patient, the TOB1 expression levels were compared with that in the corresponding noncancerous tissue. In $84.4 \%$ (76/90) of specimens, TOB1 expression in the cytoplasm was reduced in cancerous tissues compared with noncancerous tissues of the 90 pairs of gastric cancer tissues. It was noted that TOB1 expression levels were low (according to the IRS score) in $67.8 \%$ (61/90) of the clinical samples from patients with gastric cancer. As for expression in the nucleus, TOB1 expression was reduced in $45.6 \%$ (41/90) of the clinical 
Table II. Association between clinicopathological factors and cytoplasmic transducer of ErbB2. 1 expression.

\begin{tabular}{|c|c|c|c|c|}
\hline \multirow[b]{2}{*}{ Characteristic } & \multicolumn{3}{|c|}{ TOB1 expression in cytoplasm } & \multirow[b]{2}{*}{ P-value } \\
\hline & Low & High & $\chi^{2}$ & \\
\hline Gender & & & & 0.634 \\
\hline Male & 45 & 20 & 0.226 & \\
\hline Female & 16 & 9 & & \\
\hline Age (years) & & & & 0.972 \\
\hline$<60$ & 25 & 12 & 0.001 & \\
\hline$\geq 60$ & 36 & 17 & & \\
\hline Tumor size (mm) & & & & 0.809 \\
\hline$<40$ & 14 & 6 & 0.058 & \\
\hline$\geq 40$ & 47 & 23 & & \\
\hline Depth of invasion & & & & 0.017 \\
\hline pT1 & 1 & 6 & 10.211 & \\
\hline pT2 & 13 & 4 & & \\
\hline pT3 & 34 & 13 & & \\
\hline pT4 & 13 & 6 & & \\
\hline Nodal status & & & & 0.345 \\
\hline Negative & 23 & 8 & 0.891 & \\
\hline Positive & 38 & 21 & & \\
\hline Metastasis & & & & 0.577 \\
\hline M0 & 55 & 25 & 0.312 & \\
\hline M1 & 6 & 4 & & \\
\hline Differentiation grade & & & & 0.034 \\
\hline Grade I & 0 & 3 & 6.769 & \\
\hline Grade II & 34 & 16 & & \\
\hline Grade III & 27 & 10 & & \\
\hline TNM stage & & & & 0.000 \\
\hline I & 4 & 11 & 19.010 & \\
\hline II & 14 & 13 & & \\
\hline III & 35 & 3 & & \\
\hline IV & 8 & 2 & & \\
\hline
\end{tabular}

TNM, tumor-node-metastasis.

samples, compared with that of the noncancerous tissues. It was also noted that TOB1 expression levels in the nucleus were high (according to the IRS score) in 54.4\% (49/90) of the clinical samples from patients with gastric cancer (Table I).

Clinical and pathological significance of cytoplasmic TOB1 expression. The expression of TOB1 was analyzed in normal and cancerous tissues in patients with gastric cancer using immunohistochemistry and tissue microarrays. Representative examples of staining for TOB1 in normal and cancerous tissues are presented demonstrated in Fig. 2A-D. Associations between specific pathological features and TOB1 expression in the cytoplasm are shown in Table II. Reduced cytoplasmic TOB1 expression was significantly associated with depth of invasion $(\mathrm{P}=0.017)$, differentiation grade $(\mathrm{P}=0.034)$ and tumor-node-metastasis (TNM) stage $(\mathrm{P}<0.000)$. Compared with the normal tissue samples, cytoplasmic TOB1 expression was almost absent in poorly differentiated carcinomas (Fig. 3A), while weak and marked TOB1 expression levels were observed in moderately and well-differentiated gastric cancer samples, respectively (Fig. 3B and C). By contrast, nuclear expression of TOB1 exhibited no significant correlation with any pathological features (data not shown).

\section{Discussion}

TOB1 is a member of the TOB/BTG gene family, is ubiquitously expressed in the adult tissues of humans and was first identified using an ErbB2 probe to screen an expression library and detect interactions between proteins (11). TOB1 was hypothesized to be a tumor suppressor gene, based on the observations that TOB-knockout mice spontaneously develop tumors and that TOB1 expression is absent in human lung and 

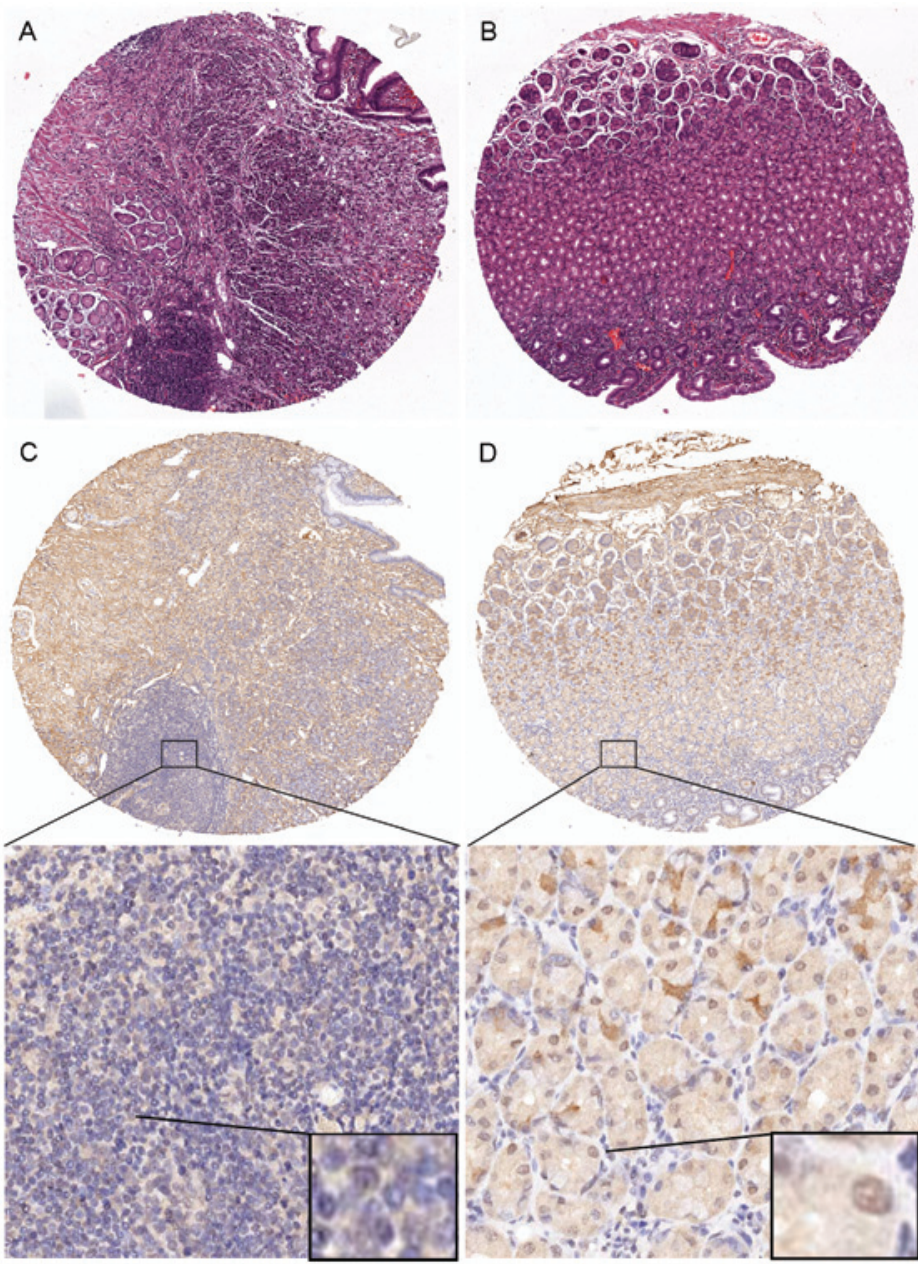

Figure 2. Expression of TOB1 in gastric cancer and adjacent normal tissues. The representative samples were obtained from the 90 microarray patients. (A) Gastric cancer tissues and (B) adjacent normal tissues were confirmed using hematoxylin and eosin staining. Immunohistochemical staining revealed (C) weak cytoplasmic TOB1 expression in gastric cancer tissues and (D) high cytoplasmic TOB1 expression in normal tissues. (A-D) Magnification, x40; (A and D) lower panels magnification, x400. TOB1, transducer of ErbB2. 1.

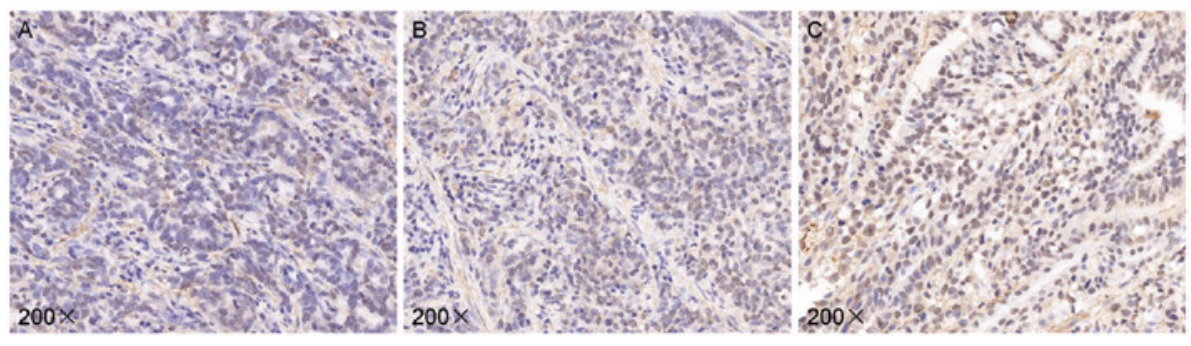

Figure 3. Immunohistochemical analysis of TOB1 expression in differentiated gastric cancer samples. (A) Cytoplasmic TOB1 expression was almost absent in patients with poorly differentiated carcinomas, while weak and marked TOB1 expression were observed in (B) moderately differentiated gastric cancer and (C) well-differentiated gastric cancer, respectively. TOB1, transducer of ErbB2. 1.

thyroid cancer (18). However, to date, the TOB1 expression pattern has not been investigated in gastric cancer (19). To the best of our knowledge, the present study is the first to report that TOB1 expression is significantly reduced in gastric cancer tissues compared with that of the adjacent normal tissues as revealed by tissue microarray analysis.

Tissue microarray analysis represents a useful tool in the investigation of large populations, and utilizes tissues routinely processed in surgical pathology (20). Tissue microarrays are widely used to evaluate the diagnostic, prognostic and therapeutic potential of novel genes. Tumor suppressor genes may negatively regulate cell proliferation, functioning in various ways to limit cell growth and proliferation (21-24). In the present study, a reduction in cytoplasmic TOB1 expression was observed in $84.4 \%$ (76/90) of gastric cancer samples compared with that of the adjacent normal tissues; furthermore, negative or low cytoplasmic expression of TOB1 was observed in $67.8 \%$ (61/90) of the human gastric cancer samples. As for nuclear TOB1 expression, no difference was identified between cancer tissues and the adjacent 
normal tissues and it exhibited no significant association with any pathological features. A significant association was found between the cytoplasmic expression levels of TOB1 and the clinicopathological characteristics of gastric cancer, including depth of invasion $(\mathrm{P}=0.017)$, differentiation grade $(\mathrm{P}=0.034)$ and TNM stage $(\mathrm{P}<0.000)$, indicating that TOB1 may be significant in angiogenesis and cell differentiation in gastric cancer.

However, in the present study, no significant correlation was identified between TOB1 expression and lymph node or distant tumor metastasis. For distant metastasis, this may be as a result of the low number of patients with gastric cancer with distant metastasis included in the present study, as patients with gastric cancer with metastasis usually do not undergo surgical resection. Previous studies have revealed that TOB1 proteins are distributed throughout the cytoplasm and nucleus (25). Notably, the subcellular distribution of TOB1 varies during cell cycle phases meaning that higher levels are detected in the cytoplasm during late $\mathrm{S}$ phase than during other phases of the cell cycle $(26,27)$. In the present study, no difference was identified in TOB1 expression between the lymph node negative and positive group. However, a significant association was identified between the expression of TOB1 and depth of invasion $(\mathrm{P}=0.017)$ and differentiation grade $(\mathrm{P}=0.034)$. In summary, with respect to lymph node metastasis, TOB1 is important in angiogenesis and cell differentiation of gastric cancer.

In conclusion, reduced TOB1 expression in gastric adenocarcinoma is associated with the extent of differentiation and the TNM stage of gastric cancer. The potential of TOB1 as a therapeutic target for gastric cancer requires further investigation.

\section{Acknowledgements}

The present study was supported by grants from the Science and Technology Program of Suzhou City (grant no. SYSD2013023) and the Social Development Project of Kunshan City (grant no. KS1224).

\section{References}

1. Siegel R, Ma J, Zou Z and Jemal A: Cancer statistics, 2014. CA Cancer J Clin 64: 9-29, 2014.

2. Zheng L, Wu C, Xi P, et al: The survival and the long-term trends of patients with gastric cancer in Shanghai, China. BMC Cancer 14: 300, 2014.

3. Ychou M, Boige V, Pignon JP, et al: Perioperative chemotherapy compared with surgery alone for resectable gastroesophageal adenocarcinoma: an FNCLCC and FFCD multicenter phase III trial. J Clin Oncol 29: 1715-1721, 2011.

4. Dikken JL, Jansen EP, Cats A, et al: Impact of the extent of surgery and postoperative chemoradiotherapy on recurrence patterns in gastric cancer. J Clin Oncol 28: 2430-2436, 2010.

5. Yan SM, Tang JJ, Huang CY, et al: Reduced expression of ZDHHC2 is associated with lymph node metastasis and poor prognosis in gastric adenocarcinoma. PLoS One 8: e56366, 2013.

6. Isobe T, Aoyagi K, Koufuji K, et al: Clinicopathological significance of hypoxia-inducible factor-1 alpha (HIF-1alpha) expression in gastric cancer. Int J Clin Oncol 18: 293-304, 2013.
7. Lee $\mathrm{J}$ and $\mathrm{Ou} \mathrm{SH}$ : Towards the goal of personalized medicine in gastric cancer-time to move beyond HER2 inhibition. Part II: Targeting gene mutations and gene amplifications and the angiogenesis pathway. Discov Med 16: 7-14, 2013.

8. Boku N: HER2-positive gastric cancer. Gastric Cancer 17: 1-12 2014.

9. Luis M, Tavares A, Carvalho LS, et al: Personalizing therapies for gastric cancer: molecular mechanisms and novel targeted therapies. World J Gastroenterol 19: 6383-6397, 2013.

10. He C, Bian XY, Ni XZ, et al: Correlation of human epidermal growth factor receptor 2 expression with clinicopathological characteristics and prognosis in gastric cancer. World $J$ Gastroenterol 19: 2171-2178, 2013.

11. Tzachanis D and Boussiotis VA: Tob, a member of the APRO family, regulates immunological quiescence and tumor suppression. Cell Cycle 8: 1019-1025, 2009.

12. Lin S, Zhu Q, Xu Y, et al: The role of the TOB1 gene in growth suppression of hepatocellular carcinoma. Oncol Lett 4: 981-987, 2012.

13. O'Malley S, Su H, Zhang T, et al: TOB suppresses breast cancer tumorigenesis. Int J Cancer 125: 1805-1813, 2009.

14. Jiao Y, Sun KK, Zhao L, et al: Suppression of human lung cancer cell proliferation and metastasis in vitro by the transducer of ErbB-2.1 (TOB1). Acta Pharmacol Sin 33: 250-260, 2012.

15. Wang XM, Li J, Yan MX, et al: Integrative analyses identify osteopontin, LAMB3 and ITGB1 as critical pro-metastatic genes for lung cancer. PLoS One 8: e55714, 2013.

16. Wang YX, Zhang XY, Zhang BF, et al: Study on the clinical significance of Argonaute2 expression in colonic carcinoma by tissue microarray. Int J Clin Exp Pathol 6: 476-484, 2013.

17. Sun Z, Wang ZN, Zhu Z, et al: Evaluation of the seventh edition of American Joint Committee on Cancer TNM staging system for gastric cancer: results from a chinese monoinstitutional study. Ann Surg Oncol 19: 1918-1927, 2012.

18. Yoshida Y, Nakamura T, Komoda M, et al: Mice lacking a transcriptional corepressor Tob are predisposed to cancer. Genes Dev 17: 1201-1206, 2003.

19. Kundu J, Wahab SM, Kundu JK, et al: Tob1 induces apoptosis and inhibits proliferation, migration and invasion of gastric cancer cells by activating Smad4 and inhibiting betacatenin signaling. Int J Oncol 41: 839-848, 2012.

20. Liu F, Cao Q, Liu N, et al: Overexpression of testes-specific protease 50 (TSP50) predicts poor prognosis in patients with gastric cancer. Gastroenterol Res Pract 2014: 498246, 2014.

21. Zhu Z, Wang J, Sun Z, et al: Flotillin2 expression correlates with HER 2 levels and poor prognosis in gastric cancer. PLoS One 8: e62365, 2013.

22. Zhou S, Xu S, Tao H, et al: CCR7 expression and intratumoral FOXP3+ regulatory $\mathrm{T}$ cells are correlated with overall survival and lymph node metastasis in gastric cancer. PLoS One 8: e74430, 2013.

23. Taubert H, Heidenreich C, Holzhausen HJ, et al: Expression of survivin detected by immunohistochemistry in the cytoplasm and in the nucleus is associated with prognosis of leiomyosarcoma and synovial sarcoma patients. BMC Cancer 10: 65, 2010.

24. Wang Q, Zhao ZB, Wang G, et al: High expression of KIF26B in breast cancer associates with poor prognosis. PLoS One 8: e61640, 2013.

25. Ezzeddine N, Chang TC, Zhu W, et al: Human TOB, an antiproliferative transcription factor, is a poly(A)-binding protein-dependent positive regulator of cytoplasmic mRNA deadenylation. Mol Cell Biol 27: 7791-7801, 2007.

26. Helms MW, Kemming D, Contag CH, et al: TOB1 is regulated by EGF-dependent HER 2 and EGFR signaling, is highly phosphorylated and indicates poor prognosis in node-negative breast cancer. Cancer Res 69: 5049-5056, 2009.

27. Yoneda M, Suzuki T, Nakamura T, et al: Deficiency of antiproliferative family protein Ana correlates with development of lung adenocarcinoma. Cancer Sci 100: 225-232, 2009. 